\title{
Excision of a Strong Markov Process
}

\author{
F. B. Knight and A.O. Pittenger
}

\section{Introduction and Definitions}

Let $\left(\Omega, \mathscr{F}, \mathscr{F}_{t}, X_{t}, \theta_{t}, P^{x}\right)$ be a strong Markov process on a locally compact space $\left(E_{\Delta}, \mathscr{E}_{\Delta}\right)$ with countable base, where $\Delta$ denotes the usual adjoined absorbing point and $\mathscr{E}_{\Delta}$ the Borel sets of $E_{\Delta}$. The definitions and notation follow those of [1]. In particular, $\mathscr{F}_{t}$ is complete in $\mathscr{F}$ relative to the family $P^{x}$, and $\zeta(\omega)=\inf \left\{t: X_{t}(\omega)=\Delta\right\}$ is the "lifetime". We assume also that for all $\omega \in \Omega$, $X_{t}(\omega)=X(t)=\omega(t)$ is right continuous for all $0 \leqq t$ and has left limits on $0<t<\zeta(\omega)$.

Now let $A_{0}$ and $B_{0}$ in $\mathscr{E}_{\Delta}$ be fixed, with (i) $\bar{A}_{0} \cap \bar{B}_{0}=\emptyset$, where $\bar{A}$ denotes the closure of $A$, and (ii) $\Delta \in B_{0}$.

The purpose of this paper is to construct a strong Markov process $Y$ from $X$ by excising or "splicing out" the round trip excursions from $A_{0}$ to $B_{0}$ back to $A_{0}$. With $A_{0}=\{\alpha\}$ and $B_{0}=\bar{B}_{0}$ this operation arose in [3] in analyzing the local time of a reflected stable process. The proof that the excised process $Y$ was again a Hunt process raised unanticipated difficulties not resolved in [3], and led to the present paper.

A second purpose of this paper is to give a concrete example of a "nonMarkovian" time change of a process which nonetheless preserves the strong Markov property. A different type of such example was given in [4] in the form $t \rightarrow t+L$, where $L$ is an exact coterminal time, e.g., the last exit from a set in $\mathscr{E}_{\Delta}$ prior to $\zeta$.

Our first observation is

Lemma 0.1. Intervals of excursion from $A_{0}$ to $B_{0}$ back to $A_{0}$ cannot accumulate before $\zeta$.

Proof. This is clear from the existence of left-hand limits up to $\zeta$ and the assumption (i).

Recalling that the passage times

$$
D_{C}=\inf \{t \geqq 0: X(t) \in C\}
$$

for $C \in \mathscr{E}_{\Delta}$ are $\mathscr{\mathscr { F }}_{t}$-stopping times [1], we next define successive random times $T_{n}^{\prime}, L_{n}$, and $T_{n}^{*}$ such that the excised excursions occur in the intervals $\left[L_{n}, T_{n}^{\prime}\right), n \geqq 1$.

Definition 0.1. With $\inf (\emptyset)=\infty$ and $\sup (\emptyset)=0$, let $T_{0}^{\prime}=D_{A_{0}}, T_{1}^{*}=D_{B_{0}}$, and $L_{1}=\sup \left\{t<T_{1}^{*}, X_{t} \in A_{0}\right\}$. Define inductively for $n \geqq 1$

$$
\begin{aligned}
T_{n}^{\prime} & =T_{n}^{*}+T_{0}^{\prime} \circ \theta_{T_{n}^{*}}, \\
T_{n+1}^{*} & =T_{n}^{\prime}+T_{1}^{*} \circ \theta_{T_{n}^{\prime}},
\end{aligned}
$$


and

$$
L_{n+1}=T_{n}^{\prime}+L_{1} \circ \theta_{T_{n}^{\prime}}
$$

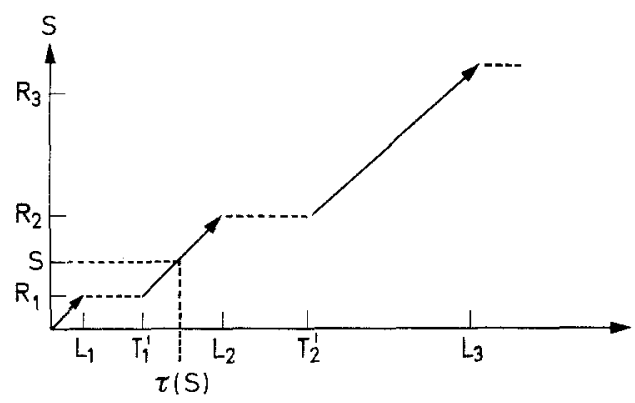

Fig. 1. Excised time $S$ and given time $\tau(S)$

Note that $T_{n}^{*}$ and $T_{n}^{\prime}$ are stopping times but $L_{n}$ is not. However, $L_{n} \in \mathscr{F}_{T_{n}^{*}}$. In the following definition, if $s$ is the time parameter of $Y$ then $N(s, \omega)$ is the number of excursions excised by time $s$ and $\tau(s)$ is corresponding time of $X$ (see Fig. 1).

Definition $0.2^{1}$. Using $\sum_{k=1}^{0}(\cdot)=0$, for $0 \leqq s<\infty$ define

$$
N(s, \omega)= \begin{cases}0 ; & 0 \leqq s<L_{1} . \\ n ; & s \in\left[L_{1}+\sum_{1}^{n-1}\left(L_{k+1}-T_{k}^{\prime}\right), L_{1}+\sum_{1}^{n}\left(L_{k+1}-T_{k}^{\prime}\right)\right), \quad T_{n}^{\prime}<\infty \\ \infty ; & \text { there is no such } n .\end{cases}
$$

Setting $S_{n}(\omega)=\sum_{1}^{n}\left(T_{k}^{\prime}-L_{k}\right)$, let

$$
\tau(s)= \begin{cases}s+S_{N(s)}(\omega) ; & N(s)<\infty \\ \infty ; & N(s)=\infty .\end{cases}
$$

The time change is plotted for a fixed $\omega$ with $0<L_{1}$ in Fig. $1^{2}$. We set $R_{n}=T_{n}^{\prime}-S_{n}$ so that $S_{n}$ represents the total deleted time prior to $T_{n}^{\prime}$, and $R_{n}$ is the total included time before $T_{n}^{\prime}$.

The following relations are consequences of the definitions:

$$
\begin{gathered}
\{\tau(0)=0\}=\left\{0<L_{1}\right\}, \\
\left\{R_{n} \leqq t<R_{n+1}\right\}=\left\{T_{n}^{\prime} \leqq \tau(t)<L_{n+1}\right\} \\
=\left\{T_{n}^{\prime} \leqq \tau(t)<T_{n+1}^{\prime}\right\}, \\
\tau(s+t)=\tau(s)+\tau(t) \circ \theta_{\tau(s)} .
\end{gathered}
$$

We can now define the excised process and its probability function.

\footnotetext{
${ }^{1}$ In definitions by cases, we use the semicolon to separate the functions from their domains of application.

${ }^{2}$ We are indebted to P.A. Meyer for this figure, and for a number of suggestions concerning our statement of the problem.
} 
Definition 0.3. For $\Lambda \in \mathscr{F}$ and $y \in E_{\Delta}$,

$$
Q^{y}(\Lambda)=\left(\begin{array}{ll}
P^{y}(\Lambda \mid \tau(0)=0) ; & P^{y}\{\tau(0)=0\}>0 \\
I_{\Lambda}\left(\omega_{x}\right) ; & \text { otherwise }
\end{array}\right.
$$

where $\omega_{x}(t)=x$ for all $t \geqq 0$ and $I_{\Lambda}$ is the indicator function of $\Lambda$.

$$
\begin{aligned}
Y_{t}(\omega)=Y(t) & = \begin{cases}X(\tau(t)) ; & P^{X(0)}\{\tau(0)=0\}>0 \\
X(0) ; & P^{X(0)}\{\tau(0)=0\}=0,\end{cases} \\
\zeta_{Y}(\omega) & =\inf \{t: Y(t)=\Delta\} .
\end{aligned}
$$

Note that $Q^{y}=P^{y}$ if $y$ is regular for $A_{0}$. We cannot assert, however, that $Q^{y}$ is $\mathscr{E}_{\Delta}$-measurable. If one wishes to obtain this property it seems to be necessary to assume

(iii) $A_{0}$ and $B_{0}$ are open or closed,

(iv) $X$ is a standard process.

We record here two propositions related to these extra assumptions.

Proposition 0.1. Under assumptions (i) through (iv), $Q^{y}\{Y(t) \in A\}$ is $\mathscr{E}_{\Delta^{-}}$-measurable for each $A \in \mathscr{E}_{\Delta}$.

Proof. If $A_{0}$ and $B_{0}$ are both open, then all of the variables in Definition 0.1 are $\mathscr{F}^{0}$-measurable, where $\mathscr{F}^{0}$ is the minimal, pre-completed $\sigma$-field, and thus setting $B_{1}=\left\{x: P^{x}\{\tau(0)=0\}=0\right\}-\{\Delta\}$ we have $B_{1} \in \mathscr{E}_{\Delta}$. If $A_{0}$ (or $B_{0}$ ) is closed, we may find, by [1, I, Corollary 10.17], a decreasing sequence of open sets whose passage times converge to that of $A_{0}$ (or $B_{0}$, respectively) $P^{x}$ almost surely for each $x$. Using these limits in place of $D_{A_{0}}$ and $D_{B_{0}}, Q^{y}$ and $B_{1}$ will be unchanged, and we can define a new process equal to $X(\tau(t))$ for all $t$ a.s. $Q^{y}$ for each $y$. Since the new process is $\mathscr{F}^{0}$-measurable, the proof is complete.

Proposition 0.2. With the notations and assumptions of Proposition 0.1, for $y \in E_{\Delta}-B_{1}$,

$$
Q^{y}\left\{Y(t) \in E_{\Delta}-B_{1} \text { for } 0 \leqq t<\infty\right\}=1 .
$$

Proof. Since $Q^{y}$ is absolutely continuous with respect to $P^{y}$ and $X$ has the strong Markov property at each $T_{n}^{\prime}$, it is enough to show that $P^{y}\left\{D_{B_{1}}<L_{1}\right\}=0$ for all $y$ in $E_{A}-B_{1}$. By [1, I, Corollary 10.17] there is an increasing sequence of compact sets $K_{n} \subset B_{1}$ such that $D_{K_{n}} \downarrow D_{B_{1}}, P^{y}$ a.s. Thus we have

$$
\begin{aligned}
P^{y}\left\{D_{B_{1}}<L_{1}\right\} & =\lim _{n \rightarrow \infty} P^{y}\left\{D_{K_{n}}<\infty, D_{K_{n}}<L_{1}\right\} \\
& \leqq \lim _{n \rightarrow \infty} E^{y}\left[D_{K_{n}}<\infty, P^{X\left(D_{K_{n}}\right)}\{\tau(0)=0\}\right] \\
& =0,
\end{aligned}
$$

since $X\left(D_{K_{n}}\right) \in B_{1}$ on $\left\{D_{K_{n}}<\infty\right\}$.

In view of Proposition 0.2, we may pose

Definition 0.4 .

$$
\theta_{t}^{Y}(\omega)= \begin{cases}\theta_{\tau(t)}(\omega) ; & X(0) \in E_{\Delta}-B_{1} \\ \omega ; & \text { otherwise. }\end{cases}
$$

Under (i)-(iv), we then have (see (0.3)) $Y_{t} \circ \theta_{s}^{Y}=Y_{s+t}$ for all $s, t \geqq 0$, except on a $Q^{(\cdot)}$-null set. 
We give three final remarks before stating the theorem. First, under assumptions (i)-(iv), it is possible that $T_{n}^{\prime}=L_{n+1}$ a.s. for all $n$ with $T_{n}^{\prime}<\infty$. In this case, one has $Q^{y}\left\{\zeta_{Y}=D_{A_{0}}\right\}=1$ for $y \in E-B_{1}$. To exclude such a degenerate case, and at the same time to insure that $P^{y}=Q^{y}$ for all $y \in A_{0}$, it suffices to assume

(v) Every point of $A_{0}$ is regular for $A_{0}$.

Indeed, this implies that $T_{n}^{\prime}<L_{n+1}$ a.s. on $\left\{T_{n}^{\prime}<\infty\right\}$ for each $n$. Second, we have $Q^{y}\{Y(0)=y\}=1$ for all $y \in E_{\Delta}$. However, the set $\left\{Y(0) \in B_{1}\right\}$ is clearly foreign to the excised process, and is retained only to avoid reducing the original state space. Third, $Y(t)$ is right-continuous, with left limits on $0<t<\zeta_{Y}$.

\section{Statement and Proof of the Theorem}

We let $\mathscr{F}_{Y}^{0}(t)$ denote the $\sigma$-field on $\Omega$ generated by $Y(s), 0 \leqq s \leqq t$, and let $\mathscr{F}_{Y}(t)$ be the usual completion of $\mathscr{F}_{Y}^{0}(t)$ in $\mathscr{F}_{Y}=\mathscr{F}_{Y}(\infty)$ with respect to $\left\{Q^{v}, y \in E_{\Delta}\right\}$.

Theorem. If $A_{0}$ and $B_{0}$ satisfy conditions (i) and (ii), then $Y=\left(\Omega, \mathscr{F}_{Y}, \mathscr{F}_{Y}(t), Y(t), Q^{y}\right)$ is a strong Markov process on $\left(E_{\Delta}, \hat{\mathscr{E}}_{\Delta}\right)$ where $\hat{\mathscr{E}}_{\Delta}$ is the collection of universally measurable sets of $E_{\Delta}$.

Corollary. If (i)-(iv) are assumed, and if $A_{0}=\{\alpha\}$ and $\alpha$ is regular for $\{\alpha\}$, then $Y=\left(\Omega, \mathscr{F}_{Y}, \mathscr{F}_{Y}(t), Y(t), \theta_{t}^{Y}, Q^{y}\right)$ is a standard process. If, moreover, $\alpha$ is recurrent (i.e. for each $N, X(t)=\alpha$ for some $t>N$ a.s.) then $Y$ is a Hunt process.

Proof of the Theorem. In view of $[1, \mathrm{I}$., Thm. 7.3] it suffices to prove the strong Markov property for $\mathscr{F}_{Y}^{0}(t+)$-stopping times $T_{Y}\left(\left\{T_{Y}<t\right\} \in \mathscr{F}_{Y}^{0}(t)\right.$ for all $\left.t\right)$. In particular, this will imply the right-continuity of $\mathscr{F}_{Y}(t)$. The key to the proof is the introduction of a sequence $T_{n}, n \geqq 0$, of $\mathscr{F}$-stopping times such that $T_{n}=\tau\left(T_{Y}\right)$ on $\left\{N\left(T_{Y}\right)=n\right\}$, and we begin with $T_{0}$.

By definition of $\mathscr{F}_{Y}^{0}(t)$, there is a Borel function $f_{t}\left(x_{1}, x_{2}, \ldots\right)$ on the usual infinite product space, and a sequence $s_{1}, s_{2}, \ldots$ with $s_{n} \leqq t$, such that

$$
I_{\left\{T_{Y}<t\right\}}=f_{t}\left(Y\left(s_{1}\right), Y\left(s_{2}\right), \ldots\right) \text {. }
$$

To simplify the notation, we suppress the dependence of the $s_{n}$ on $t$, and assume that $f_{t}$ takes only the values 0 and 1 . Note that we have

$$
T_{Y}(\omega)=\inf \left\{s: 0=\prod_{\substack{r<s \\ r \text { rational }}}\left(1-f_{r}\left(Y\left(s_{1}\right), Y\left(s_{2}\right), \ldots\right)\right)\right\} .
$$

Definition 1.1. Let $f_{t}(\omega)=f_{t}\left(X\left(s_{1}\right), X\left(s_{2}\right), \ldots\right)$ and

$$
T_{0}=\inf \left\{s: 0=\prod_{\substack{r<s \\ r \text { rational }}}\left(1-f_{r}\left(X\left(s_{1}\right), X\left(s_{2}\right), \ldots\right)\right)\right\},
$$

where the countable family $\left\{s_{i}, s_{i} \leqq r\right\}$ appearing in $f_{r}$ depends on $r$.

Lemma 1.1. $T_{0}$ is an $\mathscr{F}_{t}$-stopping time. Moreover,

$$
\left\{N\left(T_{Y}\right)=0\right\}=\left\{N\left(T_{0}\right)=0\right\} \subset\left\{T_{0}=T_{Y}\right\} .
$$

Proof. The first assertion follows from

$$
\left\{T_{0}<t\right\}=\bigcup_{\substack{r<t \\ r \text { rational }}}\left\{f_{r}(\omega)=1\right\} \in \mathscr{F}_{t} .
$$


Now if $N\left(T_{Y}\right)=0$, then for some $\varepsilon>0, X_{s}=Y_{s}$ for $0 \leqq s<T_{Y}+\varepsilon, T_{0}=T_{Y}$, and $N\left(T_{0}\right)=0$. Conversely if $N\left(T_{0}\right)=0$, there must be a hit of $A_{0}$ after time $T_{0}$ and before $D_{B_{0}}$. Hence $X(s)=Y(s)$ up to $T_{0}+\varepsilon$ for some $\varepsilon>0, T_{Y}=T_{0}$, and $N\left(T_{Y}\right)=0$.

To define $T_{n}, n \geqq 1$, we first introduce a sequence of mappings $\varphi_{n}: \Omega \rightarrow \Omega$ which "splice out" the first $n$ round trips from $A_{0}$ to $B_{0}$ back to $A_{0}$, if such exist.

Definition 1.2. For all $\omega \in \Omega$, let $\varphi_{0} \omega=\omega$ and for $n \geqq 1$

$$
\left(\varphi_{n} \omega\right)(t)= \begin{cases}\omega(\tau(t)) ; & N(t)<n \\ \omega\left(t+S_{n}\right) ; & N(t) \geqq n\end{cases}
$$

where $\omega(\infty)=\Delta$ and $S_{n}$ is as in Definition 0.2. Then letting $\Lambda_{n}=\left\{T_{0} \circ \varphi_{n}+S_{n-1} \geqq L_{n}\right\}$,

$$
T_{n}(\omega)= \begin{cases}T_{0}\left(\varphi_{n} \omega\right)+S_{n}(\omega) ; & \omega \in \Lambda_{n} \\ \infty ; & \omega \in \Lambda_{n}^{c} .\end{cases}
$$

As before we have for $n \geqq 1$

Lemma 1.2. $T_{n}$ is an $\mathscr{F}_{r}$-stopping time, and

$$
\left\{N\left(T_{Y}\right)=n\right\}=\left\{N\left(T_{0} \circ \varphi_{n}\right)=n\right\} \subset\left\{T_{n}=\tau\left(T_{Y}\right)\right\} .
$$

Proof. By adding $T_{n}^{\prime}-L_{n}$ to both sides of the inequality defining $\Lambda_{n}$ we observe that $T_{n}^{\prime} \leqq T_{n}$. An examination of the definition shows that $S_{n}$ is $\mathscr{F}_{T_{n}^{\prime}}$-measurable, and that the definition of $\Lambda_{n}$ depends on $\omega(s)$ only for $s \leqq L_{n}-S_{n-1}+S_{n}=T_{n}^{\prime}$. Hence $\Lambda_{n} \in \mathscr{F}_{T_{n}^{\prime}}$.

Since $\left\{T_{n}<t\right\}=\Lambda_{n} \cap\left\{T_{n}^{\prime}<t\right\} \cap\left\{T_{0} \circ \varphi_{n}+S_{n}<t\right\}$, it suffices to show that the last set is in $\mathscr{F}_{t}$. But this follows from the same argument-the dependence on $\omega(s)$ is only up through $\left(t-S_{n}\right)+S_{n}=t$.

Now suppose that $t$ is any time and $N(t, \omega)=n$. Then for some $\varepsilon>0$

$$
X\left(\varphi_{n} \omega(s)\right)=Y(s), \quad 0 \leqq s<t+\varepsilon .
$$

Consequently if $N\left(T_{Y}\right)=n$, then

$$
f_{r}\left(Y\left(s_{1}\right), Y\left(s_{2}\right), \ldots\right)=f_{r}\left(X\left(\varphi_{n} \omega\left(s_{1}\right)\right), X\left(\varphi_{n} \omega\left(s_{2}\right)\right), \ldots\right)
$$

for all $r<T_{Y}+\varepsilon$, some $\varepsilon>0$, and $T_{Y}=T_{0} \circ \varphi_{n}$. If $N\left(T_{0} \circ \varphi_{n}\right)=n$, the same argument applies. Since $T_{n}=T_{0} \circ \varphi_{n}+S_{n}=\tau\left(T_{Y}\right)$ on $\left\{N\left(T_{Y}\right)=n\right\}$, the proof is complete.

To prove the theorem we need one more lemma, whose proof will be postponed to follow that of the theorem itself. that

Lemma 1.3. For each $A_{0} \in \mathscr{F}_{Y}^{0}\left(T_{Y}\right)$ and each $n \geqq 0$ there exists a $\Gamma_{n} \in \mathscr{F}_{T_{n}}$ such

Granting this lemma, and replacing $\Lambda \in \mathscr{F}_{Y}\left(T_{Y}\right)$ by a $P^{y}$-equivalent $\Lambda_{0} \in \mathscr{F}_{Y}^{0}\left(T_{Y}\right)$, we have for $A \in \mathscr{E}_{\Delta}$,

$$
\begin{aligned}
P^{y}(A, & \left.\tau(0)=0, N\left(T_{Y}\right)=n ; Y\left(T_{Y}+t\right) \in A\right) \\
& =P^{y}\left(\Gamma_{n}, \tau(0)=0, N\left(T_{Y}\right) \geqq n, \tau(0) \circ \theta_{T_{n}}(\omega)=0, X\left(T_{n}+\tau(t) \circ \theta_{T_{n}}\right) \in A\right) \\
& =E^{y}\left(Q^{X\left(T_{n}\right)}(X(\tau(t)) \in A) ; \Gamma_{n}, \tau(0)=0, N\left(T_{Y}\right)=n\right) \\
& =E^{y}\left(Q^{Y\left(T_{Y}\right)}(Y(t) \in A) ; \tau(0)=0, A, N\left(T_{Y}\right)=n\right)
\end{aligned}
$$


where the second equality follows from the strong Markov property for $X$ and the definition of $Q^{y}$. Noting that

$$
\bigcup_{n=0}^{\infty}\left\{N\left(T_{Y}\right)=n\right\}=\left\{T_{Y}<\zeta_{Y}\right\}
$$

we have after a summation over $n$

$$
Q^{y}\left(\Lambda, T_{Y}<\zeta_{Y}, Y\left(T_{Y}+t\right) \in A\right)=\hat{E}^{y}\left(Q^{Y\left(T_{Y}\right)}(Y(t) \in A) ; A, T_{Y}<\zeta_{Y}\right)
$$

where $\hat{E}^{y}$ denotes the conditional expectation given $\tau(0)=0$. Since both sides are universally measurable, for any probability measure $\mu$ we can integrate to obtain

$$
Q^{\mu}\left(\Lambda, T_{Y}<\zeta_{Y}, Y\left(T_{Y}+t\right) \in A\right)=\hat{E}^{\mu}\left(Q^{Y\left(T_{Y}\right)}(Y(t) \in A) ; A, T_{Y}<\zeta_{Y}\right) .
$$

Over $\left\{T_{Y} \geqq \zeta_{Y}\right\}$ the analogous result is obvious, completing the proof of the strong Markov property.

We return to the proof of Lemma 1.3. For each $t$, let $h_{t}\left(x_{1}, x_{2}, \ldots\right)$ be a Borel function such that

$$
I_{\Lambda_{10}}(\omega) I_{\left\{T_{Y}<t\right\}}(\omega)=h_{t}\left(Y\left(s_{1}\right), Y\left(s_{2}\right), \ldots\right)
$$

where as before the countable family $\left\{s_{i}, s_{i} \leqq t\right\}$ depends on $t$ and $h_{t}$ takes on only the values 0 and 1 . Let $h_{t}(\omega)=h_{t}\left(X\left(\varphi_{n} \omega\left(s_{1}\right)\right), X\left(\varphi_{n} \omega\left(s_{2}\right)\right), \ldots\right)$, and introduce the functions

$$
k_{t}= \begin{cases}0 ; & N\left(T_{0} \circ \varphi_{n} \omega\right)<n \\ 1-\prod_{\substack{r<t \\ r \text { rational }}}\left(1-h_{r}(\omega) I_{\left\{T_{0} \circ \varphi_{n}<r\right\}}\right) ; & \text { otherwise. }\end{cases}
$$

One notes that $k_{t}$ is non-decreasing in $t$, left-continuous, and takes on only the values 0 and 1 . Moreover, it is 1 only if $T_{0} \circ \varphi_{n}<t$. To handle a technical detail, we require the rather cumbersome last restriction in the following definition. Let $\Gamma_{t}=\left\{k_{t}=1\right.$, and for all rational $r^{\prime}<r<t,\left(k_{r}-k_{r}\right) I_{\left\{T_{0}{ }^{\circ} \varphi_{n}<r^{\prime}\right\}}=0$ ? We show first that $\Gamma_{t}$ is non-decreasing in $t$, and second that $\Gamma_{n}=\lim _{t \rightarrow \infty} \Gamma_{t}$ is the sired set. Suppose, indeed, that for $s<t, \Gamma_{s}-\Gamma_{t} \neq \emptyset$. Then for $\omega \in \Gamma_{s}-\Gamma_{t}$ we have $k_{s}=k_{t}=1$, and so $\left(k_{r}-k_{r^{\prime}}\right) I_{\left\{\boldsymbol{T}_{0} \circ \varphi_{n}<\boldsymbol{r}^{\prime}\right\}}=1$ for some $r^{\prime}<r<t$. Then $k_{r^{\prime}}=0$ and hence $r^{\prime}<s$. But since $k_{t}$ is left-continuous, there is also an $r<s$ with $k_{r}=1$, contradicting $\omega \in \Gamma_{s}$. Similarly, if $\omega \in \Gamma_{n} \cap\left\{T_{0} \circ \varphi_{n}<t\right\}$ then for $s>t, \omega \in \Gamma_{s}$ implies $k_{r}=1$ for $t-\varepsilon<r<s$ for some $\varepsilon>0$, and hence $\omega \in \Gamma_{t}$. Thus $\Gamma_{n} \cap\left\{T_{0} \circ \varphi_{n}<t\right\}=\Gamma_{t}$.

To prove the lemma, we now show that

$$
A_{0} \cap\left\{N\left(T_{Y}\right)=n, T_{Y}<t, N(t)=n\right\}=\Gamma_{n} \cap\left\{N\left(T_{Y}\right)=n, T_{0} \circ \varphi_{n}<t, N(t)=n\right\}
$$

for each $t$. If $\omega$ is in the left hand side then $Y(s)=X\left(\varphi_{n} \omega(s)\right), 0 \leqq s<t$, and we have easily $k_{t}=1$ and $\omega \in \Gamma_{t} \subset \Gamma_{n}$. Together with $T_{Y}=T_{0} \circ \varphi_{n}$, this gives the inclusion from left to right. On the other hand, if $\omega \notin \Lambda_{0}$ but satisfies the other conditions on the left, then $h_{r}(\omega)=0$ for $r<t, k_{t}=0$, and $\omega \notin \Gamma_{t}$. Since $T_{0} \circ \varphi_{n}<t, \omega \notin \Gamma_{u}$ for 
$u>t$, and so $\omega \notin \Gamma_{n}$. Finally, to prove that $\Gamma_{n} \in \mathscr{F}_{T_{n}}$, we have

$$
\begin{aligned}
\Gamma_{n} \cap\left\{T_{n}<t\right\} & =\bigcup_{\substack{r<t \\
r \text { rational }}} \Gamma_{n} \cap\left\{T_{n}^{\prime}<t\right\} \cap\left\{T_{0} \circ \varphi_{n} \omega<r<t-S_{n}\right\} \\
& =\bigcup_{\substack{r<t \\
r \text { rational }}} \Gamma_{r} \cap\left\{T_{n}^{\prime}<t\right\} \cap\left\{T_{0} \circ \varphi_{n} \omega<r<t-S_{n}\right\}
\end{aligned}
$$

and simply argue as in Lemma 1.2 that the dependence on $\omega$ does not extend beyond $\left(t-S_{n}\right)+S_{n}=t$. This completes the proof of the theorem.

Proof of the Corollary. The measurability conditions and the translation operators were discussed in Proposition $0.1 \mathrm{ff}$., and we need only prove quasileft continuity on $\left(0, \zeta_{Y}\right)$. For $T_{Y} \leqq \zeta_{Y}$ let $T_{n}$ and $\left\{N\left(T_{Y}\right)=n\right\}$ correspond to $T_{Y}$ as before, let $R_{j}$ converge upward to $T_{Y}$, and let $T_{n, j}$ correspond in the same way to $R_{j}$. By $\left[1\right.$, p. 36] we may assume that $R_{j}$ and $T_{Y}$ are $\mathscr{F}_{Y}^{0}(t+)$-stopping times, and we must show that

$$
Q^{\mu}\left\{Y\left(R_{j}\right) \rightarrow Y\left(T_{Y}\right) ; T_{Y}<\zeta_{Y}\right\}=Q^{\mu}\left\{T_{Y}<\zeta_{Y}\right\} .
$$

For $\omega \in\left\{N\left(T_{Y}\right)=n, T_{Y}<\zeta_{Y}\right\}$ either $T_{n}^{\prime}<T_{n}<L_{n+1}$, or else $\tau\left(T_{Y}\right)=T_{n}^{\prime}$. In the former case, $Y\left(R_{j}\right) \rightarrow Y\left(T_{Y}\right)$ follows from $X\left(T_{n, j}\right) \rightarrow X\left(T_{n}\right)$. In the latter case, either $R_{j}=T_{Y}$ for all large $j$, or else $\tau\left(R_{j}\right)=T_{n-1, j}$ for all large $j$ a.s., and

$$
\lim _{j} Y\left(R_{j}\right)=\lim _{j} X\left(T_{n-1, j}\right)=\alpha=X\left(T_{n}^{\prime}\right)=Y\left(T_{Y}\right),
$$

since $A_{0}=\{\alpha\}$.

If $\alpha$ is recurrent, then $T_{n}^{\prime}=\infty$ implies $L_{n}=\infty$ a.s. Since $\alpha$ is regular for $\{\alpha\}$ we have $\zeta=\infty$ a.s., and also since $X\left(T_{n}^{\prime}\right)=\alpha, \sum_{k=0}^{\infty}\left(L_{k+1}-T_{k}^{\prime}\right)=\infty$ a.s. If follows that $\zeta_{Y}=\infty, Q^{\mu}$ a.s. for each $\mu$, and $Y$ is a Hunt process. This completes the proof.

\section{References}

1. Blumenthal, R.M., Getoor, R.K.: Markov processes and potential theory. New York: Academic Press 1968.

2. Courrège, P., Priouret, P.: Temps d'arrêt d'une fonction aléatoire: relations d'equivalence associées et propriétés de décomposition. Publ. Inst. Statist. Univ. Paris 14, 245-274 (1965).

3. Knight, F.B.: The local time at zero of the reflected symmetric stable process. Z. Wahrscheinlichkeitstheorie verw. Geb. 19, 180-190 (1971).

4. Meyer, P.A., Smythe, R.T., Walsh, J.B.: Birth and Death of Markov Processes. Proceedings of the Sixth Berkeley Symposium on Probability and Statistics (to appear).

F. B. Knight

Department of Mathematics

University of Illinois

Urbana, I11. 61801

USA

\author{
A.O. Pittenger \\ Department of Mathematics \\ University of Michigan \\ Ann Arbor, Michigan 48104 \\ USA
}

